\title{
The role of spinal adrenergic receptors on the antinociception of ginsenosides in a rat postoperative pain model
}

\author{
In Ji Kim ${ }^{1}$, Cheon Hee Park ${ }^{1}$, Seong Heon Lee ${ }^{2}$, and Myung Ha Yoon ${ }^{2}$ \\ Department of Anesthesiology and Pain Medicine, ${ }^{1}$ Gwangju Christian Hospital, ${ }^{2}$ Chonnam National University Medical School, \\ Gwangju, Korea
}

Background: The effect of spinal adrenergic and cholinergic receptors on the anti-nociceptive effect of intrathecal ginsenosides was determined in a rat postoperative pain model.

Methods: Catheters were placed into the intrathecal space of male Sprague-Dawley rats. Postoperative pain was evoked by an incision to the plantar surface of a hind paw. Withdrawal thresholds was used as a nociceptive parameter and was measured with a von Frey filament. After observing the effect of intrathecal ginsenosides, an alpha- 1 adrenergic receptor antagonist (prazosin), an alpha-2 adrenergic receptor antagonist (yohimbine), a muscarinic acetylcholine receptor antagonist (atropine), and a nicotinic acetylcholine receptor antagonist (mecamylamine) were given 10 min before administration of the ginsenosides to analyze the contribution of spinal adrenergic and cholinergic receptors on the antinociceptive effect of ginsenosides.

Results: Paw incision decreased withdrawal threshold in incised site of paw, but no change of withdrawal threshold was not seen in non-incised site. The intrathecal ginsenosides increased withdrawal threshold of the incised paw in a dosedependent manner. Pre-treatment with both prazosin and intrathecal yohimbine antagonized the anti-nociceptive effect of the ginsenosides. However, pre-treatments with atropine or mecamylamine had any effect on the antinociceptive activity of ginsenosides.

Conclusions: Intrathecal ginsenosides are effective in attenuation of postoperative pain induced in the rat model. Antinociceptive action of ginsenosides is partially mediated by spinal adrenergic receptors, but does not appear to be related to spinal cholinergic receptors. (Korean J Anesthesiol 2013; 65: 55-60)

Key Words: Adrenergic receptors, Ginsenosides, Intrathecal, Postoperative pain, Spinal cord.

\footnotetext{
Received: March 11, 2013. Revised: 1st, March 20, 2013; 2nd, May 5, 2013. Accepted: May 10, 2013.

Corresponding author: Myung Ha Yoon, M.D., Ph.D., Department of Anesthesiology and Pain Medicine, Chonnam National University Hospital, Hak-dong, Dong-gu, Gwangju 501-757, Korea. Tel: 82-62-220-6887, Fax: 82-62-220-6880, E-mail: mhyoon@chonnam.ac.kr

(c) This is an open-access article distributed under the terms of the Creative Commons Attribution Non-Commercial License (http:// creativecommons.org/licenses/by-nc/3.0/), which permits unrestricted non-commercial use, distribution, and reproduction in any medium, provided the original work is properly cited.
} 


\section{Introduction}

Surgical incision and intra-operative tissue injuries are a source of postoperative pain. Those surgical insults directly activate peripheral nociceptors, which in turn increase central neuronal excitabilityand lead to peripheral and central sensitization [1]. This sensitization processes results in specific pain symptoms such as hyperalgesia (an increase in pain responsiveness to painful stimuli) and allodynia (an increase in pain responsiveness to non-painful stimuli). If the sensitization process is left unchecked, a chronic state of postoperative pain may develop other comorbid symptoms such as insomnia, depression, and anxiety in postoperative patients. Moreover, these chronic postoperative pain patients can experience psychological and social disability, which significantly reduces the quality of life over time [2]. Thus, active postoperative pain management is the crucial first step in the prevention of the development of chronic pain disorder after an operation. Despite the vast amount of research on this topic, no analgesic medication is able to effectively block the sensitization process without unwanted side effects.

Traditionally, ginsenosides have been used as an herbal medicine [3]. Interestingly, spinal ginsenosides have been investigated and found to attenuate a variety of nociception in animal models [4-8]. Particularly, intrathecally-administered Korean red ginseng and ginsenosides suppress formalin-induced nociceptive behavior among rats, and the anti-nociceptive property of ginsenosides is suspectied to be mediated by both adrenergic and cholinergic receptors in the spinal cord [6-8]. It is well known that adrenergic and cholinergic systems modulate nociceptive transmission at the spinal level, which is mediated through the respective receptors $[9,10]$.

Thus, the aim of this present study was to evaluate the effect of ginsenosides in a rat postoperative pain model and to elucidate any role of adrenergic and cholinergic receptors have on the activity of ginsenosides in the spine.

\section{Materials and Methods}

\section{Animal preparation and intrathecal catheterization}

The experimental protocols were reviewed and approved by the Institutional Animal Care and Use Committee. Adult male Sprague-Dawley rats, weighing 250-300 g, were used in this study. Rats were housed in each cage of the animal facility maintained in a temperature-controlled room $\left(22 \pm 0.5^{\circ} \mathrm{C}\right)$ with $12: 12 \mathrm{~h}$ light/dark cycle. Food and water were provided ad libitum. For intrathecal catheterization, each rat was fixed in a stereotaxic apparatus under sevoflurane anesthesia, and the proximal portion of catheter was inserted into the subarachnoid space through the cisterna magna and was extended into the lumbar enlargement of the spinal cord [11]. The distal portion of catheter was secured to the top of the head and closed up with a 30-gauge wire. The skin was sutured with a 3-0 silk. After cessation of general anesthesia, fully ambulatory rats were returned to the respective individual cage in the animal facility, whereas rats demonstrating neurological impairment were immediately euthanized by volatile anesthetics. The incisional surgery of hind paw was carried out five days after the intrathecal catheterization, and behavioral studies with study medications were performed on the same day.

\section{Drugs}

The following drugs were used: ginsenosides, prazosin, yohimbine, atropine, and mecamylamine. Ginsenosides were kindly provided by the Korea Ginseng and Tobacco Research Institute (Daejon, Korea). The remaining four agents were purchased from Sigma Aldrich Co. (St. Louis, MO, USA). Ginsenosides were prepared in a solution of dimethyl sulfoxide, and the others were dissolved in normal saline solution. All agents were intrathecally administered in a $10 \mu \mathrm{l}$ solution, followed by an additional $10 \mu \mathrm{l}$ of normal saline to flush the catheter using a hand-driven, gear-operated syringe pump.

\section{Nociceptive model}

Postoperative pain induction was performed according to the incisional model described by Brennan et al. [12]. Under sevoflurane anesthesia, a $1 \mathrm{~cm}$ long incision was made through the plantar skin and fascia of the left hind paw, longitudinally, about $0.5 \mathrm{~cm}$ from the proximal edge of heel toward the toes. The plantaris muscle was elevated and incised longitudinally with the muscle origin and insertion points intact. The incised skin was sutured with a 4-0 silk. Prophylactic antibiotics were applied over the incision. Following the closure of this incision, the development of postoperative pain was examined for 360 $\min$.

\section{Postoperative pain measurements}

Paw withdrawal threshold was determined by the up and down method [13]. The hind paws were accessed via apertures created in the mesh floor of the cage. von Frey filaments (Stoelting, Wood Dale, IL, USA) of eight calibers $(0.4,0.7,1.2$, 2.0, 3.6, 5.5, 8.5, and $15.0 \mathrm{~g}$ ) were used to stimulate the plantar surface of the hind paw for $4 \mathrm{sec}$ while the hair was spread away from the incision. A positive response was defined as brisk withdrawal or paw flinching after the application of a filament. Withdrawal threshold was measured two times for each filament 
caliber, with about 3-min stimulation-free period in-between, and the average value was assigned to the determined threshold. Only rats showing allodynia (withdrawal threshold $<5 \mathrm{~g}$ ) were included in this study.

\section{Experimental design}

The total number of rats used was 70 , with $4-7$ rats per treatment group, and each rat was used only once for test. On the day of test, experimental drugs were intrathecally administered in a random style.

\section{Administration of intrathecal agents}

Before each experiment, rats were placed in individual cages and were left undisturbed for 20-30 min of acclimation period. The effects of ginsenosides $(100,300$, and 1,000 $\mu \mathrm{g})$ were examined. Withdrawal threshold was established before the incision, and this measurement was repeated at 120,150, 180,240 , and $360 \mathrm{~min}$ after incision. The withdrawal threshold measured $120 \mathrm{~min}$ after incision was regarded as the baseline post-incision threshold. The ginsenosides solution was delivered intrathecally immediately after testing at $120 \mathrm{~min}$ following incisional surgery. Next, rats were pretreated with adrenergic and cholinergic receptor antagonists to determine which receptor affected ginsenoside activity. These antagonists were administered intrathecally 10 min before delivering the intrathecal ginsenosides $(1,000 \mu \mathrm{g})$. Adrenergic and cholinergic receptor antagonists were selected based on relevant receptor affinity and selectivity, and the doses were determined by preliminary experiments designed to identify the maximum dose which did not affect the control response or motor function. The following drugs were used: alpha-1 adrenergic receptor antagonist prazosin $(3 \mu \mathrm{g})$, alpha-2 adrenergic receptor antagonist yohimbine $(10 \mu \mathrm{g})$, muscarinic acetylcholine receptor antagonist atropine $(10 \mu \mathrm{g})$, and nicotinic acetylcholine receptor antagonist mecamylamine $(10 \mu \mathrm{g})$.

\section{Statistical analysis}

Data are expressed as means \pm SEM. Time response data are presented as withdrawal thresholds in g. Dose-response data are presented as percentages of maximum possible effect (\%MPE). The withdrawal threshold data from von Frey filament testing were converted to according to the formula: \%MPE $=[$ (post-drug threshold - baseline post-incision threshold $) /$ (pre-incision threshold - baseline post-incision threshold)] $\times$ 100. Dose-response data were analyzed by one-way analysis of variance followed by Scheffés post hoc test. The difference in the withdrawal threshold between incised and non-incised sites was analyzed by paired t-test. Comparison of antagonism for the effect of ginsenosides was analyzed by unpaired t-test. $\mathrm{P}$ values $<0.05$ were considered to indicate statistical significance.

\section{Results}

Paw incision reduced the withdrawal threshold immediately following the incision, which lasted at the incised site during the observation period (Fig. 1). The withdrawal threshold at the non-incised site (the contralateral paw) was not altered by the incision (Fig. 1).

Pre-incisional withdrawal thresholds in the left and right hind paws were $14.5 \pm 0.5$ and $14.0 \pm 0.6 \mathrm{~g}$, respectively. No differences were noted on the pre-incision withdrawal threshold and baseline post-incision threshold (immediately before ginsenoside delivery) of the incised site across the whole group.

Intrathecal ginsenosides increased withdrawal threshold at the incised site in a dose-dependent fashion (Fig. 2).

Intrathecal prazosin (alpha-1 adrenergic receptor antagonist) and yohimbine (alpha-2 adrenergic receptor antagonist) reversed the anti-nociceptive effect of the ginsenosides (Fig. 3). However, intrathecal atropine (muscarinic acetylcholine receptor antagonist) and mecamylamine (nicotinic acetylcholine receptor antagonist) did not reverse the effect of the ginsenosides (Fig. 4).

Additionally, intrathecal prazosin, yohimbine, atropine, and mecamylamine alone had little or no effect on incision-induced withdrawal threshold (Fig. 3 and 4).

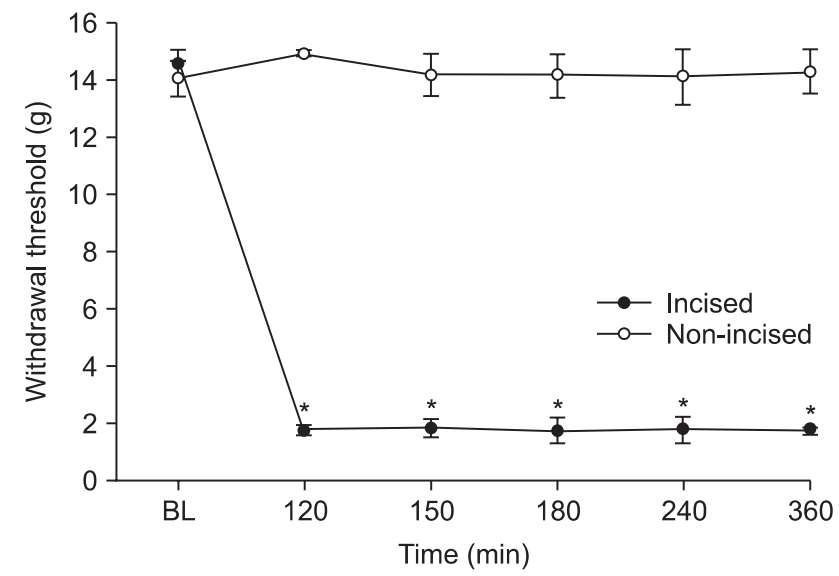

Fig. 1. Time course of the hind paw withdrawal response to von Frey filaments after paw incision. Data are presented as the withdrawal thresholds. Each line represents the mean \pm SEM of seven rats. Control data were obtained $2 \mathrm{~h}$ after incision. BL: baseline withdrawal threshold measured before paw incision. ${ }^{\star} \mathrm{P}<0.001$, significant difference between the incised site and a non-incised site. 

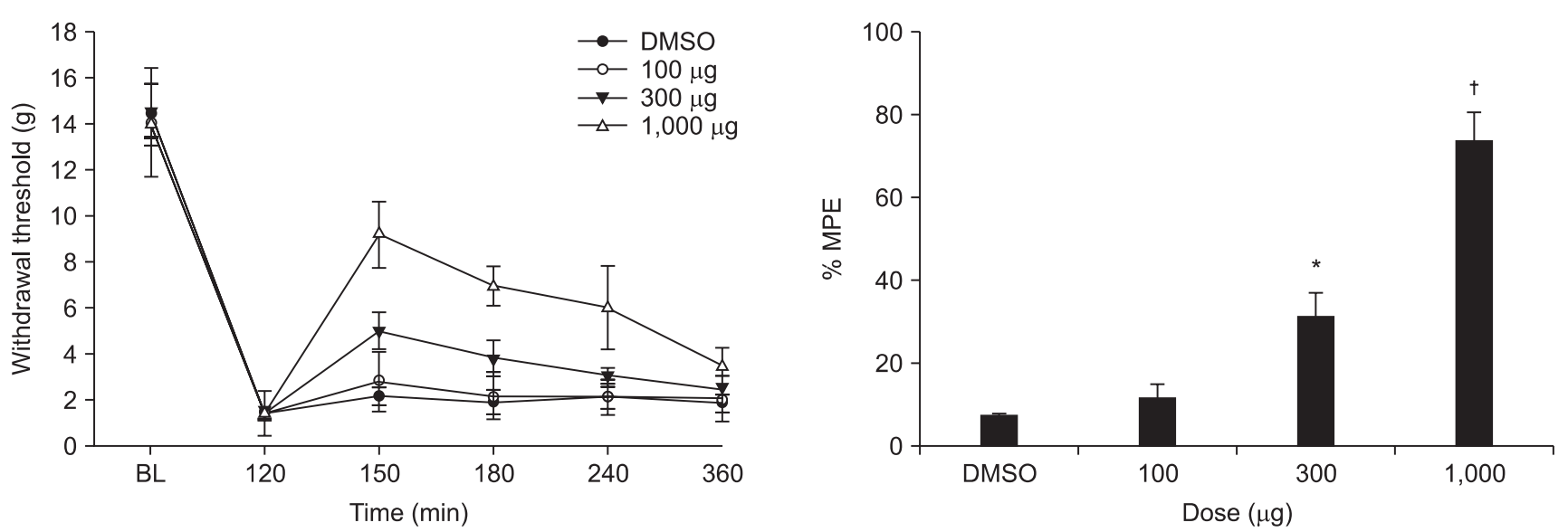

Fig. 2. Effects of intrathecal ginsenosides on hind paw withdrawal response to von Frey filaments after paw incision. Data are presented as the withdrawal thresholds or the percentages of maximal possible effect (\%MPE). Each line represents the mean \pm SEM of 5-7 rats. Ginsenosides were administered immediately after measuring the control post-incisional threshold. BL: baseline withdrawal threshold measured before paw incision, DMSO: dimethyl sulfoxide. Ginsenosides produced a dose-dependent increase in the withdrawal threshold of the incised site after paw incision, $* \mathrm{P}<$ $0.05,{ }^{\dagger} \mathrm{P}<0.001$.

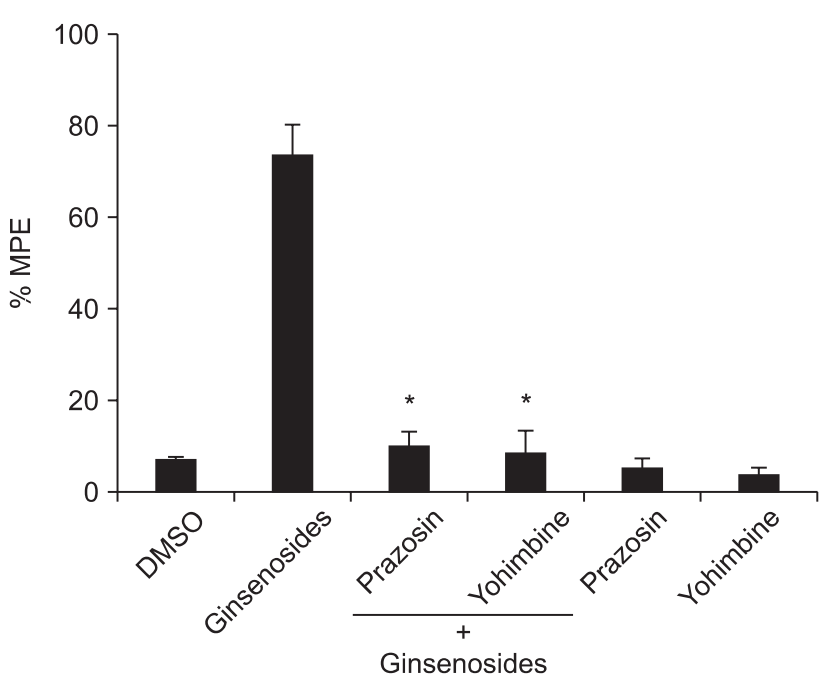

Fig. 3. Effects of intrathecal prazosin $(3 \mu \mathrm{g})$ and yohimbine $(10 \mu \mathrm{g})$ on antinociception of intrathecal ginsenosides $(1,000 \mu \mathrm{g})$. Prazosin and yohimbine were administered 10 min before the delivery of ginsenosides, and then the withdrawal threshold was measured. Data are presented as withdrawal thresholds or percentages of maximal possible effect (\%MPE). DMSO: dimethyl sulfoxide. Prazosin and yohimbine reversed the effect of the ginsenosides. Each bar represents the mean \pm SEM of $4-6$ rats. $* \mathrm{P}<$ 0.001 compared with ginsenosides.

\section{Discussion}

Despite the advancement of surgical and anesthetic techniques, postoperative pain is inevitable in most circumstances. Although efficacious analgesia is believed to improve patient recovery and decrease mortality rates after surgery [14,15], postoperative pain management is a critical issue. The cause of

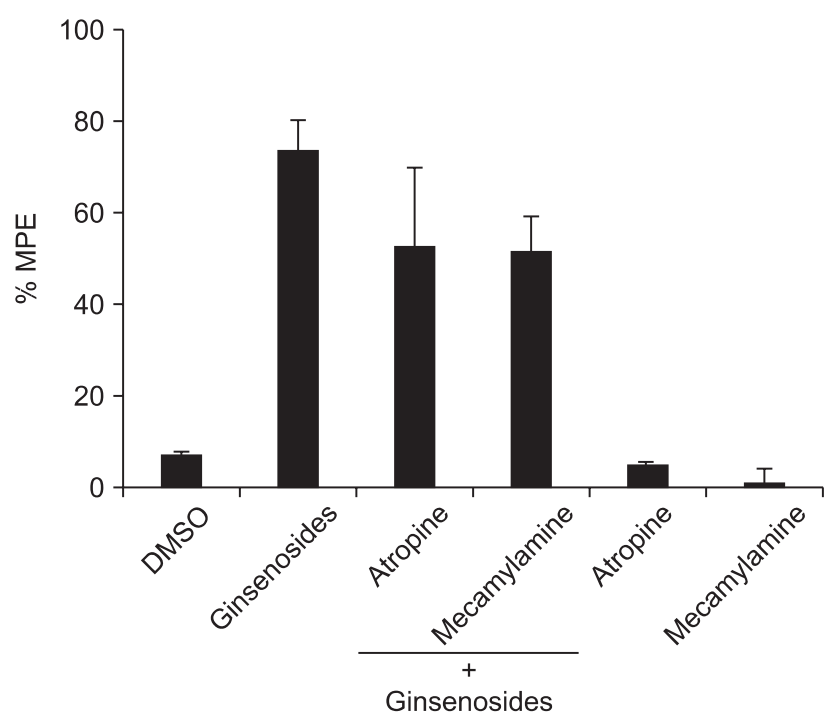

Fig. 4. The effects of intrathecal atropine $(10 \mu \mathrm{g})$ and mecamylamine $(10 \mu \mathrm{g})$ on the antinociception of intrathecal ginsenosides $(1,000 \mu \mathrm{g})$. Atropine and mecamylamine were administered $10 \mathrm{~min}$ before the delivery of ginsenosides, and the withdrawal threshold was measured. Data are presented as withdrawal thresholds or percentages of maximal possible effect (\%MPE). DMSO: dimethyl sulfoxide. Neither atropine nor mecamylamine altered the effect of the ginsenosides. Each bar represents the mean \pm SEM of $4-6$ rats.

postoperative pain can be traced back to the endogenic biologic events which take place during the operation. In the presence of operative incision, surgical manipulation, and tissue injury, the biologic response is to release proinflammatory mediators at an incision site. This response induces peripheral and central sensitization and gives rise to the phenomenon of pain [1]. Furthermore, major surgical procedures may cause not only 
pain but also decreased organ function, making a prolongation of hospital stay [16]. In addition, acute postoperative pain can persist and become chronic in nature, and this is a significant clinical problem because of the difficulties associated with chronic pain management [17]. Recent works suggest that molecular changes of genes and neuronal activity are involved in the prolongation of pain [18]. Therefore, both minimization of surgical stimulus and proper postoperative pain control may improve the outcome of postoperative patients.

To date, opioids have been the main analgesic agent used for postoperative pain management; however, they cause undesirable and often serious side effects, including nausea, vomiting, pruritis, urinary retention, and respiratory depression, which limit their clinical use [19]. Therefore, the development of new powerful analgesics that lack complications is needed. Ginseng plants have long been used in Oriental cultures for various reasons, including analgesia [3]. The pharmacologic property of ginseng plants (genus Panax) is mediated by a class of $\sim 20$ saponins which are named gisenosides after the plant [20]. These saponins have a four-ring, steroid-like chemical structure that resembles acetylcholine, adrenaline, histamine, and opioids, and the anti-nociceptive effect of ginsenosides may be relevant to these known neuro-modulators. However, the mechanism(s) of anti-nociception by gisenosides and their effective binding sites are not completely understood. Several lines of evidence indicate that ginsenosides reduce voltage-gated $\mathrm{Ca}^{2+}$ current in sensory neurons, but this effect is not blocked by alpha- 2 adrenergic, muscarinic, gamma aminobutyric acid (GABA), or opioid receptor antagonists [21]. Furthermore, the anti-nociceptive action of ginsenosides administered was not blocked by an opioid receptor antagonist during a mouse tailflick test [22]. However, previous studies have demonstrated that intrathecal ginsenosides produce an anti-nociceptive effect in the rat formalin test, which is mediated through adrenergic, cholinergic, and opioid receptors in the spinal cord [6-8]. Furthermore, intrathecal Korean red ginseng decreased postoperative pain in rats through a spinal opioid receptor but not a GABA receptor [5].

In the present study, intrathecal ginsenosides increased the withdrawal threshold to von Frey filament test after paw incision, and this finding was consistent with a previous report [5]. Thus, the results of this study suggest that ginsenosides may be useful for postoperative pain control at the spinal level.

On one hand, the anti-nociceptive effect of intrathecal ginsenosides was reversed by both intrathecal administration of the alpha-1 and alpha-2 adrenergic receptor antagonists. On the other hand, neither the muscarinic nor nicotinic receptor antagonists altered the anti-nociception of intrathecal ginsenosides. These findings suggest that, at the spinal level of pain cascade, the anti-nocieceptive action of ginsenosides may be mediated through adrenergic receptors without any involvement from cholinergic receptors. However, spinal cholinergic receptors contribute the antinociceptive effect of Korean red ginseng [6]. Although the reasons for this discrepancy are not clear, the difference in experimental methodology and, specifically, the types of stimuli may be a clue. With formalin stimulation as the pain-inducing stimulus, anti-nociception was reversed by cholinergic receptor antagonists in the previous study [6], whereas such antagonism was not noted in this study, where incisional pain model was employed. Further research on how different types of nociceptive test affects are mediated via different neural pathways is warranted.

The effects of ginsenosides on motor function was not evaluated in this study, because our previous findings indicated no motor function impairment at the highest dose $(1,000 \mu \mathrm{g})$ used in this study.

Spinal ginsenosides are not available for clinical use, but intrathecal administration of ginsenosides may be useful for management of postoperative pain in the future.

In summary, intrathecal ginsenosides decreased postoperative pain in this rat plantar incisional pain model, and alpha-1 and 2 adrenergic receptors appear to be involved in antinociception mechanism of gisenosides along the spinal sensory tract.

\section{References}

1. Woolf CJ, Chong MS. Preemptive analgesia--treating postoperative pain by preventing the establishment of central sensitization. Anesth Analg 1993; 77: 362-79.

2. Lerner RK, Esterhai JL Jr, Polomono RC, Cheatle MC, Heppenstall RB, Brighton CT. Psychosocial, functional, and quality of life assessment of patients with posttraumatic fracture nonunion, chronic refractory osteomyelitis, and lower extremity amputation. Arch Phys Med Rehabil 1991; 72: 122-6.

3. Liu CX, Xiao PG. Recent advances on ginseng research in China. J Ethnopharmacol 1992; 36: 27-38.

4. Choi SS, Han EJ, Han KJ, Lee HK, Suh HW. Antinociceptive effects of ginsenosides injected intracerebroventricularly or intrathecally in substance P-induced pain model. Planta Med 2003; 69: 1001-4.

5. Shin DJ, Yoon MH, Lee HG, Kim WM, Park BY, Kim YO, et al. The effect of treatment with intrathecal ginsenosides in a rat model of postoperative pain. Korean J Pain 2007; 20: 100-5. 
6. Kim SY, Yoon MH, Lee HG, Kim WM, Lee JD, Kim YO, et al. The role of adrenergic and cholinergic receptors on the antinociception of Korean red ginseng in the spinal cord of rats. Korean J Pain 2008; 21: 27-32.

7. Yoon MH, Huang LJ, Choi JI, Lee HG, Kim WM, Kim CM. Antinociceptive effect of intrathecal ginsenosides through alpha-2 adrenoceptors in the formalin test of rats. Br J Anaesth 2011; 106: 371-9.

8. Yoon MH, Kim KS, Lee HG, Kim CM, Kim WM, Choi JI, et al. Synergistic interaction between intrathecal ginsenosides and morphine on formalin-induced nociception in rats. J Pain 2011; 12: 774-81.

9. Pertovaara A. Noradrenergic pain modulation. Prog Neurobiol 2006; 80: 53-83

10. Jones PG, Dunlop J. Targeting the cholinergic system as a therapeutic strategy for the treatment of pain. Neuropharmacology 2007; 53: 197-206.

11. Yaksh TL, Rudy TA. Chronic catheterization of the spinal subarachnoid space. Physiol Behav 1976; 17: 1031-6.

12. Brennan TJ, Vandermeulen EP, Gebhart GF. Characterization of a rat model of incisional pain. Pain 1996; 64: 493-501.

13. Chaplan SR, Bach FW, Pogrel JW, Chung JM, Yaksh TL. Quantitative assessment of tactile allodynia in the rat paw. J Neurosci Methods 1994; 53: 55-63.

14. Scott NB, Kehlet H. Regional anaesthesia and surgical morbidity. Br J Surg 1988; 75: 299-304.

15. Yeager MP, Glass DD, Neff RK, Brinck-Johnsen T. Epidural anesthesia and analgesia in high-risk surgical patients. Anesthesiology 1987; 66: 729-36.

16. Kehlet H, Holte K. Effect of postoperative analgesia on surgical outcome. Br J Anaesth 2001; 87: 62-72.

17. Macrae WA. Chronic pain after surgery. Br J Anaesth 2001; 87: 88-98.

18. Ji RR, Woolf CJ. Neuronal plasticity and signal transduction in nociceptive neurons: implications for the initiation and maintenance of pathological pain. Neurobiol Dis 2001; 8: 1-10.

19. White MJ, Berghausen EJ, Dumont SW, Tsueda K, Schroeder JA, Vogel RL, et al. Side effects during continuous epidural infusion of morphine and fentanyl. Can J Anaesth 1992; 39: 576-82.

20. Attele AS, Wu JA, Yuan CS. Ginseng pharmacology: multiple constituents and multiple actions. Biochem Pharmacol 1999; 58: $1685-93$.

21. Nah SY, McCleskey EW. Ginseng root extract inhibits calcium channels in rat sensory neurons through a similar path, but different receptor, as mutype opioids. J Ethnopharmacol 1994; 42: 45-51.

22. Shin YH, Jung OM, Nah JJ, Nam KY, Kim CY, Nah SY. Ginsenosides that produce differential antinociception in mice. Gen Pharmacol 1999; 32: 653-9. 